\title{
Defectos de la pared abdominal. Estudio comparativo entre onfalocele y gastrosquisis
}

\author{
JULIO NAZER H. ${ }^{1}$, LUCÍA CIFUENTES O. ${ }^{2}$, ALFREDO AGUILA R. ${ }^{1}$ \\ 1. Médico Pediatra, Maternidad Hospital Clínico Universidad de Chile. \\ 2. Médico, Instituto de Ciencias Biomédicas, Facultad de Medicina, Universidad de Chile.
}

\begin{abstract}
Abdominal wall defects. A Comparative investigation between omphalocele and gastroschisis

Introduction: Omphalocele and Gastroschisis are the two most common congenital abdominal wall (AWD) defects. Objectives: To determine birth prevalence of Omphalocele and Gastroaquisis; to verify variations in different periods and to study the associated morbidity, mortality and survival. Patients and Method: The database of the Latin American Collaborative Study of Congenital Malformations (ECLAMC) was searched between the years 1996 and 2010. Results: 33 newborns (NB) with AWD were investigated (11.6 per 10,000 births). 19 of the cases corresponded to omphalocele (6.7 per 10,000 births) and 14 to gastroschisis ( 4.9 per 10,000 births). Children with omphalocele had significantly greater association with other malformations and the frequency in males was higher and showed higher mortality rates than gastroschisis. All children with gastroschisis were born alive; they were predominantly female infants whose mothers were significantly younger. Conclusion: Birth omphalocele and gastroschisis prevalence were higher than published information, perhaps due to the fact that the HCUCH (Clinical Hospital of Universidad de Chile) is a reference center. Both anomalies showed a significant increase in their birth prevalence rates when compared with those previously obtained by us in the same hospital. Significant differences between these two entities were described. Omphalocele presented higher stillbirth, mortality and fatality rates; higher frequency of associated malformations and higher average maternal age.

(Key words: Abdominal wall defects, omphalocele, gastroschisis, congenital malformation).

Rev Chil Pediatr 2013; 84 (4): 403-408
\end{abstract}

\section{RESUMEN}

Introducción: Onfalocele y gastrosquisis son los defectos de la pared abdominal (DPA) más frecuentes, que por su importancia y las diferencias entre ellos merecen ser estudiados en forma especial. Objetivos: Establecer las tasas de prevalencia de onfalocele y gastroaquisis al nacimiento. Verificar variaciones de ellas en dis-

Recibido el 03 de enero de 2012, devuelto para corregir el 23 de agosto de 2012, segunda versión 20 de noviembre de 2012 , tercera versión 19 de marzo de 2013, aceptado para publicación el 15 de abril de 2013.

Este trabajo cumple con los requisitos sobre consentimiento /asentimiento informado, comité de ética, financiamiento, estudios animales y sobre la ausencia de conflictos de intereses según corresponda.

Correspondencia a:

Dr. Julio Nazer H.

E-mail: jnazer@redclinicauchile.cl 
tintos períodos. Estudiar la morbimortalidad asociada y su sobrevida. Pacientes y Método: Se revisó la base de datos ECLAMC (Estudio Colaborativo Latino Americano de Malformaciones Congénitas) para el período 1996-2010. Resultados: Se pesquizarón 33 recién nacidos (RN) con DPA. (11,6 por 10.000 nacimientos). De ellos 19 fueron onfalocele $(6,7$ por 10.000$)$ y 14 (4,9 por 10.000) gastrosquisis. Los niños con onfalocele tenían significativamente mayor asociación con otras malformaciones, su frecuencia en el sexo masculino fue mayor y mostró mayor letalidad que la gastrosquisis. Todos los niños con gastrosquisis nacieron vivos, con predominio del sexo femenino, sus madres eran significativamente más jóvenes. Conclusión: Onfalocele y gastrosquisis presentaron una prevalencia al nacimiento mayor que lo publicado, probablemente por ser el HCUCH un Centro de Referencia. Ambas anomalías mostraron un aumento significativo en sus tasas de prevalencia al nacimiento al compararlas con las obtenidas anteriormente por nosotros en el mismo hospital. Se demostró diferencias significativas entre estas dos entidades: Onfalocele tiene mayor mortinatalidad, mortalidad y letalidad, mayor frecuencia de malformaciones asociadas y mayor promedio de edad materna.

(Palabras clave: Defectos de pared abdominal, onfalocele, gastrosquisis, malformación congénita).

Rev Chil Pediatr 2013; 84 (4): 403-408

\section{Introducción}

El onfalocele y la gastrosquisis son los defectos de la pared abdominal (DPA), que por su frecuencia, importancia y diferencias entre ellos, conviene estudiar detenidamente. Otros, como la Pentalogía de Cantrell, muy poco frecuente, por su baja prevalencia no hace variar la tasa del grupo total.

El onfalocele se define como un defecto de la pared abdominal, de tamaño variable, que se ubica en directa relación con el cordón umbilical, que contiene asas intestinales y a veces otros órganos como parte del hígado, recubiertos por amnios en la superficie externa, peritoneo en la superficie interna, y entre ambos gelatina de Wharton, junto a ellos los vasos propios del cordón.

La etiopatogenia del onfalocele ha sido explicada como un cierre incompleto de los pliegues laterales del embrión durante la cuarta semana de gestación, secundaria a un defecto del desarrollo del mesoderma a nivel del saco vitelino, que hace que la mayor parte de los órganos abdominales permanezcan fuera del embrión dentro de un saco transparente formado por el amnios, peritoneo parietal y gelatina de Wharton ${ }^{1-3}$. Ello ocurre a la sexta semana de gestación. Al terminar su formación, alrededor de la $10^{\circ}$ a la $12^{\circ}$ semana, el intestino regresa a la cavidad, rota y se fija a la pared posterior. Cualquier alteración de este proceso puede producir defectos de cierre de ella ${ }^{4-6}$.
$\mathrm{Su}$ incidencia se estima entre 1,5 y 3 por 10.000 nacimientos $^{7}$. La tasa de prevalencia al nacimiento en Chile fue de 2,7 por 10.000 nacimientos durante el período 1982-2003 ${ }^{8}$. La tasa en el HCUCH, para el período 1991-2001 fue de 6,28 por $10.000^{9}$.

La gastrosquisis es un defecto de la pared abdominal anterior, paraumbilical habitualmente a derecha, por el que salen las asas intestinales las que flotan libremente en el líquido amniótico, sólo recubiertas por el peritoneo visceral.

$\mathrm{Su}$ etiopatogenia corresponde a probablemente una disrupción vascular a nivel de la arteria onfalomesentérica derecha y de la vena umbilical derecha que en un momento de su embriogénesis involucionan ${ }^{10-12}$. Si el desarrollo y la involución ordenados se alteran tanto en su intensidad como en la cronología, se produciría un defecto de la pared abdominal como efecto de una isquemia de la pared ${ }^{11,12}$. Ha sido relacionada con la edad materna baja, como también con exposiciones a drogas, tabaco y alcohol $^{13-15}$.

$\mathrm{Su}$ prevalencia al nacimiento se estima entre 0,4 y 3 por 10.000 nacimientos $^{15}$. En la revisión del período 1991-2001 en el $\mathrm{HCUCH}$ fue de 2,1 por $10.000^{7}$.

Otra diferencia importante entre estas dos entidades es el pronóstico, debido a que el riesgo que tienen de presentar malformaciones asociadas es muy diferente ${ }^{1,4}$. La gastrosquisis se presenta asociada a otras malformaciones 
con una frecuencia entre un 10 y $20 \%$, generalmente son del sistema gastrointestinal, como atresia intestinal, vólvulos y menos frecuentemente, duplicaciones intestinales y divertículo de Meckel ${ }^{1,4,16}$. En el onfalocele, por el contrario, la asociación con otras anomalías es muy alta $^{1,8,9,16}$. Como es el caso de las anomalías cromosómicas como trisomía 13, 18 y 21 que pueden presentarse hasta en un $30 \%$ de los pacientes ${ }^{16}$. Las cardiopatías son frecuentes en estos pacientes, se pueden presentar entre 30 a $50 \%$ de los niños afectados ${ }^{17}$. Con frecuencia hay asociación con malformaciones múltiples muchas veces conformando síndromes, como el Beckwith-Wiedemann, que se caracteriza por onfalocele, macrosomía, macroglosia, hipoglicemia neonatal precoz producida por hiperinsulinismo debido a hiperplasia del páncreas $^{1,17}$, con una alta frecuencia de mortinatos.

Los objetivos de este estudio son: Estimar las tasas de prevalencia al nacimiento de onfalocele y gastrosquisis en la maternidad del $\mathrm{HCUCH}$, verificar si se han producido variaciones de ellas al compararlas con períodos anteriores publicados por nosotros, estudiar la mortalidad y letalidad producida por estas patologías. Comprobar la frecuencia con que se presentan en cada grupo etario de las madres, e individualizar las diferencias demográficas entre estas dos entidades.

\section{Pacientes y Método}

La maternidad del Hospital Clínico de la Universidad de Chile (HCUCH) es miembro desde 1969 del Estudio Latino Americano de Malformaciones Congénitas (ECLAMC) y lleva un registro de todos los recién nacidos, vivos y mortinatos que pesan al nacer $500 \mathrm{~g}$ o más y que presenten una o más malformaciones congénitas.

En este estudio recurrimos a la base de datos del ECLAMC y estudiamos todos los nacimientos ocurridos entre enero de 1996 y diciembre de 2010 en la maternidad del HCUCH. Se determinaron la tasa de prevalencia al nacimiento de onfalocele y gastrosquisis, y se compararon con las obtenidas en estudios previos, por nuestro mismo grupo, con el fin de evaluar posibles variaciones.
Se analizó la mortalidad asociada y la sobrevida de ellas.

Se estudió la distribución etaria de las madres, así como las diferencias de algunas variables demográficas, como edad materna, edad gestacional, peso de nacimiento, consumo de alcohol, drogas y tabaquismo, en un intento por caracterizar las condiciones asociadas a ambas entidades.

Se retiró de la muestra un caso de Pentalogía de Cantrell (0,4/10.000).

El estudio estadístico se realizó comparando promedios entre los dos grupos (onfalocele, gastrosquisis) mediante prueba $t$ de student $y$ las variables categóricas se compararon con prueba exacta de Fisher.

\section{Resultados}

Entre enero de 1996 y diciembre de 2010 ocurrieron 28.544 nacimientos consecutivos, 28.316 nacidos vivos (NV) y 228 mortinatos (NM), lo que representa una mortinatalidad o mortalidad fetal tardía de $0,8 \%$.

En esta muestra se encontraron $2.408 \mathrm{RN}$ portadores de una o más malformaciones congénitas, es decir una tasa de prevalencia al nacimiento de $8,4 \%$ de RN portadores de malformaciones congénitas (MFC). De ellos, 2.356 $(8,3 \%)$ eran NV y $52(0,2 \%) \mathrm{MN}$.

Entre los RN malformados se encontró 33 (11,6 por 10.000 mil nacimientos) portadores de un defecto de la pared abdominal, $25(8,8$ por $10.000 ; 25 / 28.316)$ eran NV y $8(3,5 \%$; 8/228) MN. En este grupo, 19 (6,7 por 10.000 nacimientos) presentaban onfalocele y $14 \mathrm{RN}$ gastrosquisis (4,9 por 10.000 nacimientos).

De los 19 RN con onfalocele, 8 fueron mortinatos $(42,1 \%) .5$ de los $11 \mathrm{NV}$ fallecieron antes del alta $(45,5 \%)$ lo que representa una letalidad (NV fallecidos $+\mathrm{MN}$ ) de 68,4\%.

En nuestra comunicación anterior ${ }^{4}$ la mortalidad por onfalocele fue $64,3 \%(9 / 14 \mathrm{NV})$. la letalidad fue de un 73,7\% (14/19). Todos los $\mathrm{RN}$ con gastrosquisis nacieron vivos y fueron dados de alta vivos.

La presencia de malformaciones asociadas fue significativamente más frecuente en los casos de onfalocele $(89,5 \%)$ que en los casos 
Tabla 1. Comparación entre onfalocele y gastrosquisis

\begin{tabular}{|c|c|c|c|c|c|}
\hline \multirow{2}{*}{\multicolumn{2}{|c|}{ Antecedente }} & \multicolumn{2}{|c|}{ Onfalocele } & \multicolumn{2}{|c|}{ Gastrosquisis } \\
\hline & & $n=19$ & DE & $n=14$ & DE \\
\hline \multicolumn{2}{|c|}{ Promedio peso nacimiento (g) } & 2.260 & $1.103,2$ & 2.660 & 496,6 \\
\hline \multicolumn{2}{|c|}{ Promedio edad materna (años) } & $35,4^{*}$ & 4,9 & 22,7 & 3,1 \\
\hline \multirow[t]{2}{*}{ Sexo } & Masculino & 11 & & 5 & \\
\hline & Femenino & 8 & & 9 & \\
\hline \multicolumn{2}{|c|}{ Promedio edad gestacional } & 34,9 & 4,3 & 36,7 & 1,5 \\
\hline \multicolumn{2}{|c|}{ Promedio días de hospitalización } & 22,6 & 48,2 & 62,8 & 129,2 \\
\hline \multicolumn{2}{|c|}{ Mortinatos } & $8^{*}$ & & 0 & \\
\hline \multicolumn{2}{|c|}{ NV fallecidos } & $5^{*}$ & & 0 & \\
\hline \multicolumn{2}{|c|}{ MF en la familia } & 3 & & 3 & \\
\hline \multicolumn{2}{|c|}{ MF asociadas } & $17 / 19^{*}$ & $7 / 14$ & & \\
\hline \multicolumn{2}{|c|}{ Trisomía 13 y 18} & $11^{*}$ & & 0 & \\
\hline \multicolumn{2}{|c|}{ Letalidad } & $13^{*}$ & & 0 & \\
\hline
\end{tabular}

de gastrosquisis $(57,1 \%)(\mathrm{p}=0,041) ;(17 / 19$; $89,5 \%$ vs $8 / 14 ; 57,1 \%$; $(\mathrm{p}=0,041)$.

En 8 casos de gastrosquisis había MF asociadas, 4 casos de cardiopatía congénita no cianótica, 1 de arteria umbilical unica, 1 malrotación intestinal y 2 niños con polidactilia.

Sólo 2 casos de onfalocele se presentaron en forma aislada, formando parte de un síndrome en 12 casos: 6 trisomía 18, 5 trisomía 13, 1 síndrome de Beckmann-W.

En 3 casos de onfalocele $(3 / 19 ; 15,8 \%)$ y 3 de gastrosquisis $(3 / 14 ; 21,4 \%)$ había otro MF en la familia.

En 8 casos de onfalocele $(8 / 19 ; 42,1 \%)$ y en 3 casos de gastrosquisis $(21,4 \%)$. Existía el antecedente de enfermedad aguda durante el embarazo, como infección urinaria o gripe y de enfermedades crónicas de la madre, como hipertensión arterial crónica, diabetes o hipotiroidismo, 5 casos en onfalocele $(5 / 19 ; 26,3 \%)$, y sólo 2 en gastrosquisis $(2 / 14 ; 14,3 \%)$.

El promedio de días de hospitalización fue de 22,6 días en onfalocele, 62,9 días en gastrosquisis (tabla 1) lo cual no alcanzó significancia estadística $(p=0,22)$. El peso promedio de todos los RN con defecto de la pared abdominal fue de $2.419 \mathrm{~g}, 2.260 \mathrm{~g}$ para onfalocele, con un rango entre 420 y $3.820 \mathrm{~g}$ y $2.660 \mathrm{~g}$ para gastrosquisis, con un rango entre 1.650 y 3.290 $\mathrm{g}$, estas diferencias no alcanzaron la significancia estadística $(\mathrm{p}=0,15)$. El promedio global de edad materna fue de 30 años con un rango entre 18 y 44 años. Promedio de EM en onfalocele: 35,4 años. Gastrosquisis: 22,7 años. Las edades maternas promedio de los dos grupos fueron estadísticamente diferentes entre ellos $(p=0,001)$, como se aprecia en la tabla 4 . El promedio global de edad gestacional fue de 35,7 semanas. El promedio para onfalocele fue de 34,9 semanas con un rango entre 25 y 40 semanas. Para gastrosquisis 36,7 , con un rango entre 34 y 38 semanas $(p=0,11)$.

El diagnóstico prenatal correcto se hizo en 32 de los casos de DPA. La excepción fue un niño al que se le diagnosticó prenatalmente onfalocele, en circunstancia que presentaba gastrosquisis comprobado clínica y quirúrgicamente. Además tenía una malrotación intestinal.

Al examinar la distribución por sexos de los $\mathrm{RN}$ que presentaron DPA encontramos $17 \mathrm{fe}-$ meninos y 16 masculinos. En onfalocele había 11 masculinos y 8 femeninos; en gastrosquisis 5 masculinos y 9 femeninos, sin embargo, estas diferencias no alcanzaron significación estadística $(\mathrm{p}>0,05)($ tabla 1$)$. 


\section{Discusión}

La tasa de prevalencia al nacimiento de onfalocele obtenida en esta revisión, 6,7 por 10.000 nacimientos, es significativamente mayor a la encontrada en el período 1969-1990: 1,76 por 10.000 nacimientos $(p<000,1)$. Lo mismo ocurre con gastrosquisis aumentó en forma significativa, de 0,08 por 10.000 nacimientos en el período 1969-1990 a 4,9 por 10.000 en la revisión actual $\mathrm{p}<0,0001)$. Esto no está de acuerdo con lo publicado por Curry y Rankin ${ }^{17,19}$ quienes han visto que mientras onfalocele permanece estable, gastrosquisis tiene una tendencia a aumentar.

Otro hecho demostrado y que concuerda con lo publicado por otros autores es que gastrosquisis es más frecuente en hijos de madres jóvenes y onfalocele en hijos de mujeres de mayor edad ${ }^{19-21}$. En nuestra muestra encontramos un promedio de 22,7 años en gastrosquisis contra 35,4 años en madres de niños con onfalocele, diferencia significativa $(\mathrm{p}<0,0001)$ (tabla 4). Murphy y cols. ${ }^{22}$, encontraron en una muestra de más de 120.000 nacimientos una incidencia global de 3,6 por diez mil NV; en adolescentes 12,5 por 10.000 y 2,6 por 10.000 en el resto de las mujeres. Torf y $\operatorname{cols}^{23}$, en California comunicaron que el riesgo de tener un hijo con gastrosquisis es 5 veces mayor en las mujeres jóvenes que en grupo de 25 años o más, que es 4 veces mayor entre la mujeres que consumen drogas como marihuana, anfetaminas o alcohol; que el $33 \%$ de los casos de gastrosquisis son explicados por la presencia de factores de riesgo como ser joven, consumir drogas o marginación socio-económica.

Una diferencia importante entre onfalocele y gastrosquisis es la presencia de malformaciones asociadas. Esto ocurrió en el 57,1\% de los casos de gastrosquisis y en el $89,5 \%$ de los casos de onfalocele $(\mathrm{p}=0,041)$. Las malformaciones asociadas fueron: arteria umbilical unica, polidactilia y malrotación intestinal.

Onfalocele se encontró como componente de un síndrome en 12 casos $(12 / 19,63,2 \%)$, trisomía $18(6 / 12,50 \%)$, trisomía $13(5 / 12$, $41,7 \%)$, síndrome de Beckmann-W (1/12, $8,3 \%)$.

La mortalidad es otra diferencia fundamen- tal. Entre los pacientes con gastrosquis no hubo ni mortinatos ni fallecidos, todos los RN fueron dados de alta vivos. Mientras que entre los niños con onfalocele hubo $42,1 \%$ de nacidos muertos y de los nacidos vivos fallecieron el 45,5\% de ellos (letalidad: 68,4\%). Al comparar estas cifras con lo encontrado en el período 1982-2005, observamos que la mortalidad por gastrosquisis siguió siendo $0 \%$, mientras que por onfalocele bajó de $64,3 \%(9 / 14)$ a $45,5 \%$ $(5 / 11)$ y la letalidad de $73,7 \%(14 / 19)$ a $68,4 \%$ 13/19) $(\mathrm{p}<0,0001)$.

Estos hallazgos los consideramos muy importantes desde el punto de vista médico-social, ya que contribuye, en parte, a disminuir la mortalidad infantil por malformaciones congénitas, gracias seguramente al mejor diagnóstico prenatal, que permite seleccionar el hospital con infraestructura adecuada para dar la atención integral del paciente. Por supuesto este mismo hecho hace que por ser el $\mathrm{HCUCH}$ un Centro de Referencia, la prevalencia de estas patologías al nacimiento sean mayores que en el resto de los hospitales.

La mortalidad y la letalidad de estas dos anomalías congénitas las hace completamente diferentes, especialmente en el manejo médico antenatal, en el parto y período neonatal. El estudio prolijo de las posibles malformaciones asociadas puede proporcionar datos que mejoren la sobrevida a través de las soluciones quirúrgicas oportunas de ellas.

El núnero total relativamente bajo de casos de DPA es también una limitante al momento de sacar conclusiones.

La diferencia encontrada en el promedio de días de estada en el Servicio entre onfalocele (39 días) y gastrosquisis (62,8 días) se explica por que 3 de los 5 niños NV con onfalocele fallecieron a los pocos minutos u horas de nacido, los otros dos fallecieron a los 2 y 3 días respectivamente, lo que hace bajar considerablemente el promedio del total de días de hospitalización.

En cuanto a la distribución por sexos de los $\mathrm{RN}$ afectados se encontró que el sexo masculino predominaba en los niños con onfalocele (11/8) el sexo femenino en los RN con gastrosquis (9/5). Sin embargo, estas diferencias no alcanzaron significación estadística $(\mathrm{p}>0,05)$. 


\section{Conclusiones}

Onfalocele y gastrosquisis en nuestra muestra presentaron una prevalencia al nacimiento mayor que lo publicado por otros autores. Ambas anomalías mostraron, además, un aumento significativo en sus tasas al compararlas con las obtenidas anteriormente por nosotros en el mismo hospital.

Desde el punto de vista clínico encontramos diferencias significativas entre estas dos entidades: onfalocele tiene mayor mortinatalidad, mortalidad y letalidad. Mayor frecuencia de malformaciones asociadas. Mayor severidad de estas anomalías asociadas. Mayor promedio de edad materna.

\section{Referencias}

1.- Larsen $W$ : Embriología humana. Madrid 23 Edición. Elsevier Science. 2003.

2.- Langman J: Medical Embriology. En: Digestive Tube and its derivatives. Baltimore, Williams and Wilkins, 1972.

3.- Vermeij-Keers $C$, Hartwig $N G$, van $\operatorname{der}$ Werff JF: Embryonic develoment of ventral body wall and its congenital malformations. Semin Pediatr Surg 1996; 5 (2): 82-9.

4.- Ledbetter DJ: Gastrosquisis y Onfalocele Sur Clin N Am 2006; 86: 249-60.

5.- Muñoz P, Rodríguez A, Valdés $V$, et al: Defectos de la pared anterior del abdomen: Diagnóstico prenatal y seguimiwento. Rev Chil Ultrasonog 2006; 9: 72-9.

6.- Lynn BC: Trastornos del cordon umbilical, del uraco y del conducto onfalomesentérico. En Tratado de Neonatología de Avery. Séptima Edición. Ed Harcourt. Edición en español. Madrid. 2000.

7.- Congenital Malformations Worldwide: A Report from the International Clearinghouse for Birth Defects Monitoring Systems. Elsevier SCIENCE Publishers B.V. 1991; p. 139-52.

8.- Nazer J, Cifuentes L, Aguila A, Bello MP, Correa F, Melibosky R: Prevalencia de defectos de la pared abdominal al nacer. Estudio ECLAMC 1982-2005. Rev Chil Pediatr 2006; 77 (5): 481-6.

9.- Nazer J, Juárez ME, Hubner ME, Antolini M, Cifuentes L: Malformaciones congénitas del Sistema Digestivo. Maternidad del Hospital Clínico de la Universidad de Chile. Período 1991-2001. Rev Med Chile 2003; 131: 190-6.

10.- Lubinsky MS: Asociation of prenatal vascukar disrup- tions with decreased maternal age. Am J of Medical Genetics 1997; 69: 237-9.

11.- De Vries PA: The pathogenesis of gastrosquisis and onphalocele. J Pediatr Surgery 1980; 15: 245-51.

12.- Hoyme HE, Jones MC, Jones KL: Gastroschisis: abdominal wall disruption secondary to early gestational interruption of the onphalomesenteric artery. Semin perintol 1983; 7: 294-8.

13.- Yang P, Beaty TH, Khoury MJ, Chee E, Steward W, Gordis L: Genetic-epidemiologic study of onphalocele and gastrroschisis: Evidence of heterogeneity. Am J Med Genet 1992; 44: 668-75.

14.- Centres for desease Control: Congenital Malformations surveillance. U.S. department of Health and Human Service. Teratology 1993; 48: 545-709.

15.- Molik KA, Gingalewski $C A$, West $K W$ : Gastrosquisis: a plea for risk categorization. J Pediatr Surg 2001; 36 (1): 51-5.

16.- Nicolaides KH, Snijders RJ, Cheng HH: Fetal gastrointestinal and abdominal wall defects. Associated malformations and chromosomal abnormalities. Fetal Diagn Ther 1992; 7 (2): 102-15.

17.- Curry JI, McKinney P, Thornton JG: The aetiology of gastroschisis. Br. J Obstet Gynecol 2000; 107 (11): 1339-46.

18.- Nazer J, Jarpa C, Cifuentes L: Incidencia y asociaciones de arteria umbilical única em recién nacidos. Maternidad del Hospital Clínico de la Universidad de Chile. Período 1998-2010. Chil Obstet Ginecol 2011; 76 (1): 21-5.

19.- Rankin J, Dillon E, Writh C: Congenital anterior wall defects in the north of England, 1986-1996: occurrence and outcome. Prenat Diagn 1999; 19 (7): 662-8.

20.- Lubinsky MS, Torfs C, Bateson T: Lower age specific rates for gastroschisis in California blacks than in whites: an unexpected relationship of race and decreased maternal age effects in a population bases study. Abstract: American Society of Human genetics, 45 Annual meeting, Mineapolis, Minneosoita. Am. J Hum Genet 1995; 57: 318

21.- Pennam DG, Fisher RM, Noblett HR, Soothill PW: Increase in incidence of gastroschisis in de south west of England in 1995. Br J Obstet Gynecol 1998; 105: $328-31$.

22.- Murphy A, Hernández A, Finkell G: Is teen pregnangy a risk factor for abdominal wall defects (AWDS)?. Am J of Obstet and Gynecol 2005; 193: 81.

23.- Torf CP, Velie EM, Oeschli FW, Bateson TF, Curry CJR: A population-based study of gastroschisis: demographic, pregnancy \& lifestyle risk factors 1994; 54: 84-92. 